\title{
Seeing red: Relearning to read in a case of Balint's Syndrome
}

$\underline{\text { Rose, Anita }}{ }^{1}$; Wilson, Barbara $A^{2}$; Manolov , Rumen ${ }^{3}$ and Florschutz, Gerhard ${ }^{4}$

${ }^{1}$ Department of Neuropsychology, Raphael Medical Centre, Tonbridge, Kent, UK

${ }^{2}$ The Oliver Zangwill Centre for Neuropsychological Rehabilitation, Ely, UK

${ }^{3}$ Department of Behavioural Sciences Methods, University of Barcelona, Spain

${ }^{4}$ Director at Raphael Medical Centre, Tonbridge, Kent, UK

\begin{abstract}
Background and aims: Balint's Syndrome is a rare condition, often associated with hypoxic brain damage. The major characteristic is an inability to localise objects in space, another is simultanagnosia frequently resulting in reading difficulties. We present $\mathrm{RN}$, a 37 year old woman whose major problem with reading was her inability to recognise individual letters correctly in either lower or upper case. We noted, however, that she was better if the letters were shown in red type. The aims were to determine if $\mathrm{RN}$ could relearn letters of the alphabet, investigate whether colour affected her ability to learn, and to explore more specifically whether the red type also helped her to read words.
\end{abstract}

Method: Using a single case experimental ABA design, we first determined that the optimal font for RN was size 16. In the baseline (A) phase, we assessed her ability to read all lower and upper case letters of the alphabet in black ink. In the intervention (B) phase we used font size 16 in red ink and an errorless learning approach to teaching the letters. Sessions ran 5 times per week (20 minutes per session). The intervention was then applied to picture recognition and word reading with four sets of 10 words and corresponding pictures.

Results: A consistent difference was noted between initial baseline and intervention. Improvement carried over when we returned to baseline.

Conclusion: Using red type and an errorless learning approach enabled RN to re-learn letters of the alphabet and read words she was previously unable to read. This did not however generalise to her everyday life.

Keywords: Balint's Syndrome, Hypoxia, Acquired Dyslexia, Reading 
Balint's Syndrome is a rare condition, often associated with hypoxic brain damage. Balint (1909) described the syndrome as a psychic paralysis of visual fixation. There are three main indications of this condition typically seen in patients. The first is that of a fixed gaze (ocular apraxia). This is seen as an inability to scan the entire visual field despite the patient presenting with normal eye movement, intact visual fields, and intact reflex motility. The second clinical feature is optic ataxia. Patients with optic ataxia present with an inability to integrate visual and hand coordination (Ayuso-Peralta et al., 1994). The third feature which, arguably, is considered the crux of Balint's syndrome, is simultanagnosia, which Thaiss and DeBleser (1992) described as “impaired simultaneous perception". This is noted as reduced attentional field leading to the patient only being able to interpret one object or part of an object at any one time. It is this clinical feature that frequently results in reading difficulties (Balint, 1909; Humphrey et al., 1994; Karnath \& Zihl, 2003; Rafal, 1996; Rizzo \& Vecera, 2002; Shalev \& Humphreys, 2002).

There is scant information on the rehabilitation of Balint's syndrome although a few studies exist. Zihl and Kennard (1996) describe three patients in the acute stage. Intensive practice in localisation, touching and scanning was provided and attempts made to improve oculomotor functions, enlarge the field of attention and re-establish visual-spatial orientation. Minimal improvement was noted in visual exploration and fixation but there was no recovery of the spatial disorder. Looking at more functional rehabilitation, Al-Khawaja and Haboubi (2001) worked with a 41-year-old man with Balint's Syndrome following a bilateral stroke. Treatment involved practising functional tasks that increased in complexity over time. The patient developed strategies such as tactile feedback to help him direct his gaze to objects. Again, it is difficult to know whether passage of time or intervention helped but it seems likely that giving the patient confidence to engage in everyday activities facilitated the development of one or more compensatory strategies.

Gillen and Dutton (2003) worked with a 10-year-old boy with Balint's Syndrome. He had sustained meningitis when he was three years old. The first step was educational, helping the boy and his parents to understand and acknowledge his problems. He was encouraged to use his strengths and abilities to compensate for his difficulties and to ask others to provide him with information in a way that it was easiest for him to assimilate. He was helped to keep his environment uncluttered (e.g. leaving out only one pair of shoes) and to use hearing, touch, and proprioception to locate objects instead of relying primarily on vision. One year later, 
although he had not improved on objective testing, he had learned to cope better with his disability in that his performance at school, his self-esteem, his confidence and his behavioural problems had all improved (see also Toyokura \& Koike, 2006).

Wilson (2009a) spent several months working with Malcolm, a man who developed Balint's Syndrome following anoxic damage. The initial step again involved explaining to Malcolm and care staff the challenging, subtle nature of his problems. This information was repeated in a letter to be passed to new acquaintances. Three months were spent unsuccessfully trying to improve his ability to localise objects. He was then helped to compensate either by presenting one object at a time on his wheelchair tray or by making objects more distinguishable (he was much better at choosing one object from another if they maximally differed in colour, size or shape).

These studies show that improvements are difficult but possible for patients with Balint's Syndrome, especially when the interventions are tailored to deal with their specific problems. In that sense, using single-case experimental designs (SCEDs) is warranted in this field, whereas the use of appropriate SCEDs (Kratochwill et al., 2010; Tate et al., 2013) can be helpful for establishing cause-effect relations. Considering the rehabilitation process for patients with Balint's Syndrome, it would make sense that the focus of intervention is based on the main characteristics of the syndrome; the improvement of visual scanning, the development of integrated visual and hand co-ordination, and the improvement of the integration of visual elements.

The overarching aim of this study was to explore whether a cognitive rehabilitative approach using an errorless learning approach and compensatory strategies for visual impairment could improve the ability to recognise single letters and be integrated words with matching pictures thus improving reading in a patient with Balint's Syndrome.

\section{Method}

\section{Participant}

We present RN, a 37 year old woman who was attending a neurorehabilitation hospital 5 days a week for therapeutic intervention. $\mathrm{RN}$ sustained an acquired brain injury as a result of complications following the birth of her second child. RN has a diagnosis of Balint's Syndrome and Apperceptive Agnosia. She had been referred to Neuropsychology for 
assessment and intervention during which time she reported her main desire was to be able to read to her children. During the assessment it was noted her major problem with reading was her inability to recognise individual letters correctly in either lower or upper case. We noted, however, that she was better if the letters were shown in red type. The aims were to determine if RN could relearn letters of the alphabet, investigate whether colour affected her ability to learn, and if the red type also helped her to read words. A subsidiary aim was to determine whether learning to recognise single words might help in supporting her recognition of visually presented objects in the form of pictures.

\section{Design and Procedure}

Using a single-case experimental ABA design, we first determined that the optimal font for RN was size 16. In the baseline (A) phase, we assessed her ability to read all lower and upper case letters of the alphabet in black ink. In the intervention (B) phase we used font size 16 in red ink and an errorless learning approach to teaching the letters. Errorless learning is a teaching technique whereby people are prevented, as far as possible, from making mistakes while they are learning a new skill or new information. This can be carried out in a number of ways such as providing spoken or written instructions or guiding the person through a task. The principle is to minimise the possibility of erroneous responses. The reason for this is that in order to benefit from our mistakes (trial-and-error learning), we need to be able to remember those mistakes. People with memory problems have difficulty remembering their errors and, therefore, cannot correct them. Thus, erroneous responses may be strengthened or reinforced (Wilson, 2009b). Sessions ran 5 times per week (20 minutes per session). The intervention was then applied to word reading with 4 sets of words that had meaning to her and her environment. Following the reading phase we implemented the same intervention with pictures of the learned words both in a single presentation i.e. only picture and then in a matching task i.e. picture and corresponding word.

Each phase utilised a single case experimental ABA design and errorless learning, and consisted of 5 sessions per week.Each intervention also included a follow up phase at approximately 3 months. However by this stage RN's attendance had reduced to a weekly session so the follow up was completed over 2-3 sessions.

\section{Results}




\section{Comparing the Initial Baseline to the Intervention Phase}

The first step in single-case data analysis is visual inspection, due to the amount of useful information it provides about several data features, as well as its usefulness for guiding the analysis and the validation of its results (Parker, Cryer, \& Byrns, 2006). The initial visual inspection of the data suggested that most initial baselines were stable, with some exceptions (i.e., the errors for upper case letters), and that practically all intervention phase data shown a progressive improvement, that is, a downward trend. Taking these data characteristics into account, we decided to use analytical techniques that quantify both the change in level and in slope, while they also account for initial baseline trend whenever it is present - piecewise regression (Center, Casey, \& Skiba, 1985-1986) and the slope and level change procedure (SLC; Solanas, Manolov, \& Onghena, 2010). We decided to use both techniques instead of choosing one due to two reasons: (1) the computations are performed in a different way: on the basis of least squares estimation in piecewise regression versus using differencing and computing average differences in SLC; and (2) the changes in level are conceptually different: immediate change in the first intervention phase measurement occasion is quantified by piecewise regression versus average change in level (once change in slope is controlled for). The results of using these analytical techniques are presented in Table 1 and will be commented alongside visual analysis.

\section{INSERT TABLE 1 ABOUT HERE}

Regarding the alternatives for data analysis, an option for obtaining overall estimates for the immediate intervention effect and change in slope, as well as empirical Bayes individual estimates, would have been to use multilevel models (Moeyaert, Ferron, Beretvas, \& Van den Noortgate, 2014). However, we did not perform such an analysis, given that for most of the $\mathrm{A}_{1}$-B comparisons the amount of measurements available was smaller than the smallest series length for which evidence on this technique's performance is available (Ferron, Bell, Hess, Rendina-Gobioff, \& Hibbard, 2009). Moreover, it is not clear that it conceptually makes sense to use a two-level model with a single participant for whom several different outcomes are measured longitudinally.

Looking at the number of errors committed with lower case letters (Figure 1; constructed using the SCDA plug-in for R; Bulté \& Onghena, 2012), the intervention is associated with an immediate improvement (3.91 errors less according to piecewise regression; net level 
change -4.58 according to SLC) and the number of errors is gradually reduced in subsequent measurement occasions during the intervention phase $(-0.76$ less per measurement occasion according to piecewise regression and -0.64 according to SLC). For upper case letters, the intervention is associated with an immediate improvement (2.20 errors less according to piecewise regression; net level change -0.57 according to SLC). However, the improvement is less steep ( 0.70 according to piecewise regression and 1.05 according to SLC) during the intervention phase that during baseline. In general, the intervention is apparently effective, but to a greater degree of lower case letters.

\section{INSERT FIGURE 1 ABOUT HERE}

Similar change in performance is observed when looking at words (column 1 in Figure 2) referring to animals and food. However, the effect for food is larger: immediate reduction of 3.2 vs. 1.7 according to piecewise regression; net level change of 3.3 vs. 1.75 according to SLC. For words referring to instruments and utensils, what is clear is the change in slope (e.g., -2.9 according to piecewise regression; -3.0 according to SLC for instruments). However, the ordinary least squares trend lines superimposed on the graph should be interpreted with caution for the baseline phases, as with only two values it is actually not meaningful to talk about trends. In general, the intervention is effective for words as well, given that by the end of the intervention practically no errors are committed.

\section{INSERT FIGURE 2 ABOUT HERE}

Regarding pictures (column 2 in Figure 2), the data for pictures for animals do not clearly suggest an improvement, as the baseline trend seems to continue without changes into the intervention phase (an immediate increase of 1.1errors according to piecewise regression and average deterioration of 1.25 errors according to SLC, but a steeper improvement). For pictures for food, a clear effect can be seen, both in terms of an immediate change and a change in slope (an approximate reduction of 3.20-3.30 errors per measurement occasion according to both procedures). The data patterns for pictures for instruments and utensils are similar, suggesting a gradual improvement (i.e., a change in slope of approximately 1.5 errors less per data point). 
Finally, regarding the matching between words and pictures (column 3 in Figure 2), for animals and food the data patterns are similar: an initial improving baseline trend gets steeper (e.g., -1.3 according to piecewise regression and -1.5 according to SLC for animals) indicating a faster improvement than in absence of intervention. For instruments, the intervention phase trend fitted does not represent well the data, as can be seen from column 3 in Figure 2, but at least the quantifications of slope change indicate the fact that a reduction in errors is observed. For utensils, it is clearer that there both a change in level and a not very steep change in slope ( -0.4 according to piecewise regression and -0.25 according to SLC). In general, there is an improvement associated with the intervention, although errors are not completely eliminated.

\section{Comparing the Initial Baseline to the Second Baseline and the Follow Up Phase}

For the comparison between the initial baseline and the remaining conditions in which intervention was no longer provided, we decided not to choose piecewise regression or SLC, as the application of both is meaningful only when adjacent phases are compared, given that the first phase's trend is compared to the subsequent phase's trend and projections in nonadjacent phases are not warranted. An option for obtaining overall estimates for the difference between, on the one hand, the initial baseline $\left(A_{1}\right)$ and the second baseline $\left(A_{2}\right)$ and, on the other hand, the initial baseline $\left(\mathrm{A}_{1}\right)$ and the follow up (FU) was to use the recently proposed $d$-statistic (Hedges, Pustejovsky, \& Shadish, 2012, 2013). Nevertheless, we decided not to use this index, given that the amount of measurements being compared (2-3 per phase) is too small for such a procedure developed on the basis of large-sample statistical theory. Moreover, the conditions compared showed little variability, as visible from Figures 1 and 2, and thus the use of simple indicators such as the arithmetic mean and the median is justified.

The fact that the second baseline scores represent an improvement compared to the initial baseline (an average reduction ranging from 6 to 13 errors less) and, at least visually, show no important difference from the preceding intervention phase, suggests that the intervention effect is enduring. The comparison between follow up and initial baseline shows the number of errors is smaller at later stages (i.e., a better performance: an average reduction ranging from 3 to 10 errors less), but this performance achieved during intervention phase and second baseline is deteriorated for most outcomes. All means and mean differences between conditions are available from the first author upon request. 


\section{Discussion}

This article describes a single-case study of a 37 year old woman who presented with Balint's Syndrome and its core features of 1) failure to localise objects in space (optic apraxia), 2) inability to voluntarily look into the peripheral field (optic ataxia) and 3) despite adequate visual acuity unable to process more than one stimulus at a time (simultanagnosia. This occurred following an acquired brain injury as a result of complications following the birth of her second child. The patient, RN, during a neuropsychological assessment, reported that her main goal was to be able to read.

Given RN's desire to relearn to read, the reported rehabilitation programme commenced. It consisted of visuoperceptual training together with an errorless learning approach. Her rehabilitation programme was individualised following the recognition of appropriate font size and colour of letters, in this case red.

The initial phase of the programme took into consideration that patients with Balint's only recognise one object or part of an object at a time. Therefore $\mathrm{RN}$ was taught to recognise individual letters of the alphabet both in lower and upper case. The results indicate that the intervention was effective. It was noted that she was able to read upper case more easily than lower case letters.

Following RN successfully learning individual letters, the next phase of the intervention was to engage integration of these into individual words. Word sets were developed using categories pertinent to RN's environment. Once again results indicate efficacy of the intervention for 3 of the 4 categories. Given RN's goal was to be able to read, a further intervention phase of matching visual cues (i.e., pictures) to words was commenced. Similar results were obtained with significant improvement being noted in 3 of the 4 sets. At follow up the results indicate that the effects of the intervention were enduring. Overall, the results suggest it is reasonable to propose that the rehabilitation protocol used was effective.

RN's goal was to be able to re-learn to read. Whilst she was able to learn to read both single letters and category based words, generalisation to her everyday life was not noted. RN is still unable to read a book or newspaper and does not appear to be able to notice the words she has learnt in the context of a sentence. 
The main strength of this study is that it recognised the need that the rehabilitation of visual field disorders is often idiosyncratic and highly individualised and has to be tailored to the patient's unique combination of deficits and preserved functions as well as to the functional impairment that is to be rehabilitated. In the case of RN developing the intervention around specific font size and colour had a clear positive impact and also considering categories relevant to her life arguably increased compliance and motivation.

When considering limitations of the study it could be argued the current study could have been performed using a multiple-baseline design across behaviours, in this case, across types of words. The staggered implementation of the intervention for each type of words would have provided a stronger experimental evidence for the causal effect of the intervention. This is especially so, given that in the $A_{2}$ phase of the ABA design we did not expect the behaviour to return to $A_{1}$ levels; $A_{2}$ was rather used to explore whether the effects of the intervention are maintained. Practical considerations, in particular change of staff, precluded this as it would have elongated each stage.

\section{Conclusion}

Whilst we conclude that the intervention had a positive result it is clear that in providing rehabilitation for patients with visuoperceptual deficits such as Balint's syndrome a more holistic approach needs to be considered. Whilst reducing the already impoverished attentional visual field to single letters/words in order to support RN's relearning, her ability to flexibly shift attention from one level of processing to another i.e. single object to complex multi objects remains impaired. RN finds this outcome frustrating. Not considering the 3 clinical features of the condition together is arguably the main weakness of this study. This issue needs to be considered in future interventions for both $\mathrm{RN}$ and other similar patients.

\section{References}

Al-Khawaja, I., \& Haboubi, N. H. J. (2001). Neurovisual rehabilitation in Balint's Syndrome. Journal of Neurology, Neurosurgery, and Psychiatry, 70, 416. 
Ayuso-Peralta, L., Jiménez-Jiménez, F. J., Tejeiro, J., Vaquero, A., Cabrera-Valdivia, F., Madero, S., ...., García- Albea, E. (1994). Progressive multifocal leukoencephalopathy in HIV infection presenting as Balint's syndrome. Neurology, 44, 1339-1340.

Balint, R. (1909). Seelahmung des “Schauens”, optishce Ataxie, raumliche Storung der Aufmerkasmkeit [Psychic paralysis of gaze, optic ataxia, and spatial disorder of attention]. Monatschrift für Psychiatrie und Neurologie, 25, 5-81.

Baylis, G. C., Driver, J., Baylis, L. L., \& Rafal, R. D. (1994). Reading of letters and words in a patient with Balint's syndrome. Neuropsychologia, 32, 1273-1286.

Bulté, I., \& Onghena, P. (2012). When the truth hits you between the eyes: A software tool for the visual analysis of single-case experimental data. Methodology, 8, 104-114.

Center, B. A., Skiba, R. J., \& Casey, A. (1985-1986). A methodology for the quantitative synthesis of intra-subject design research. The Journal of Special Education, 19, 387-400.

Ferron, J. M., Bell, B. A., Hess, M. R., Rendina-Gobioff, G., \& Hibbard, S. T. (2009). Making treatment effect inferences from multiple-baseline data: The utility of multilevel modelling approaches. Behaviour Research Methods, 41, 372-384.

Gillen J. A., \& Dutton, G. N. (2003) Balint's Syndrome in a 10-year-old male. Developmental Medicine \& Child Neurology, 45, 349-352.

Hedges, L. V., Pustejovsky, J. E., \& Shadish, W. R. (2012). A standardized mean difference effect size for single case designs. Research Synthesis Methods, 3, 224-239.

Hedges, L. V., Pustejovsky, J. E., \& Shadish, W. R. (2013). A standardized mean difference effect size for multiple baseline designs across individuals. Research Synthesis Methods, 4, 324-341.

Humphrey, G. K., Goodale, M. A., Jakobson, L. S., \& Servos, P. (1994). The role of surface information in object recognition: Studies of a visual form agnosic and normal subjects. Perception, 23, 1457-1481.

Karnath, H.-O., \& Zihl, J. (2003). Disorders of spatial orientation. In T. Brandt, L. R. Caplan, J. Dichgans, H. C. Diener, \& C. Kennard (Eds.), Neurological disorders: Course and treatment (2nd Ed.) (pp. 277-286). San Diego, CA: Academic Press. 
Kratochwill, T. R., Hitchcock, J., Horner, R. H., Levin, J. R., Odom, S. L., Rindskopf, D. M., \& Shadish, W. R. (2010). Single-case designs technical documentation. Retrieved from What Works Clearinghouse website: http://ies.ed.gov/ncee/wwc/pdf/wwc_scd.pdf

Moeyaert, M., Ferron, J., Beretvas, S., \& Van Den Noortgate, W. (2014). From a single-level analysis to a multilevel analysis of since-case experimental designs. Journal of School Psychology, 52, 191-211.

Parker, R. I., Cryer, J., \& Byrns, G. (2006). Controlling baseline trend in single-case research. School Psychology Quarterly, 21, 418-443.

Rafal, R. (1996). Balint's syndrome In T. E. Feinberg and M. J. Farah (Eds.), Behavioural neurology and neuropsychology (pp. 233-238). New York, NY: McGraw-Hill.

Rizzo, M., \& Vecera, S. P. (2002). Psychoanatomical substrates of Balint's syndrome. Journal of Neurology, Neurosurgery and Psychiatry, 72, 162-178.

Shalev, L., \& Humphreys, G. W. (2002). Implicit location encoding via stored representations of familiar objects: Neuropsychological evidence. Cognitive Neuropsychology, 19, 721-744.

Solanas, A., Manolov, R., \& Onghena, P. (2010). Estimating slope and level change in $\mathrm{N}=1$ designs. Behavior Modification, 34, 195-218.

Tate, R. L., Perdices, M., Rosenkoetter, U., Wakima, D., Godbee, K., Togher, L., \& McDonald, S. (2013). Revision of a method quality rating scale for single-case experimental designs and n-of-1 trials: The 15-item Risk of Bias in N-of-1 Trials (RoBiNT) Scale. Neuropsychological Rehabilitation, 23, 619-638.

Thaiss, L., \& De Bleser, R. (1992). Visual agnosia: a case of reduced attentional "spotlights". Cortex, 28, 601-621.

Wilson, B. A. (2009a). Malcolm: Coping with the effects of Balint's Syndrome and topographical disorientation. In B. A. Wilson , J. J. Evans, F. Gracey, \& A. Bateman (Eds.), Neuropsychological rehabilitation: Theory, models, therapy and outcomes (pp. 304-316). Cambridge, UK: Cambridge University Press.

Wilson, B. A. (2009b). Memory rehabilitation: Integrating theory and practice. New York, NY: Guilford Press.

Zihl, J., \& Kennard, C. (1996). Disorders of higher visual functions. In T. Brandt, L. R. Caplan, J. Dichgans, H. C. Diener, \& C. Kennard (Eds.), Neurological disorders, course and treatment (pp. 201-212). San Diego, CA: Academic Press. 
Table 1. Results for the $A_{1} B$ comparison obtained using piecewise regression (Center, Skiba, \& Casey, 1985-1986) and the Slope and level change procedure (SLC; Solanas, Manolov, \& Onghena, 2010).

\begin{tabular}{lcccc}
\hline Data & $\begin{array}{l}\text { Regression } \\
\text { immediate change }\end{array}$ & $\begin{array}{l}\text { Regression } \\
\text { change in slope }\end{array}$ & $\begin{array}{l}\text { SLC change } \\
\text { in slope }\end{array}$ & $\begin{array}{l}\text { SLC net } \\
\text { change in level }\end{array}$ \\
\hline Errors lower case & -3.91 & -0.76 & -0.64 & -4.58 \\
Errors upper case & -2.20 & 0.70 & 1.05 & -0.57 \\
Words: Animals & -1.70 & -1.70 & -1.67 & -1.75 \\
Words: Food & -3.20 & -0.80 & -0.75 & -3.30 \\
Words: Instruments & -3.00 & -2.90 & -3.00 & -2.80 \\
Words: Utensils & -1.20 & -2.10 & -2.00 & -1.40 \\
Pictures: Animals & 1.10 & -0.40 & -0.50 & 1.25 \\
Pictures: Food & -3.20 & -0.80 & -0.75 & -3.30 \\
Pictures: Instruments & 0.86 & -1.70 & -1.50 & 0.47 \\
Pictures: Utensils & 0.00 & -1.30 & -1.25 & -0.10 \\
Matching: Animals & -1.97 & -1.30 & -1.50 & -1.67 \\
Matching: Food & 0.53 & -1.00 & -0.75 & 0.03 \\
Matching: Instruments & -0.20 & -0.80 & -1.00 & 0.20 \\
Matching: Utensils & -2.20 & -0.40 & -0.25 & -2.50 \\
\hline
\end{tabular}




\section{Errors for upper case letters}
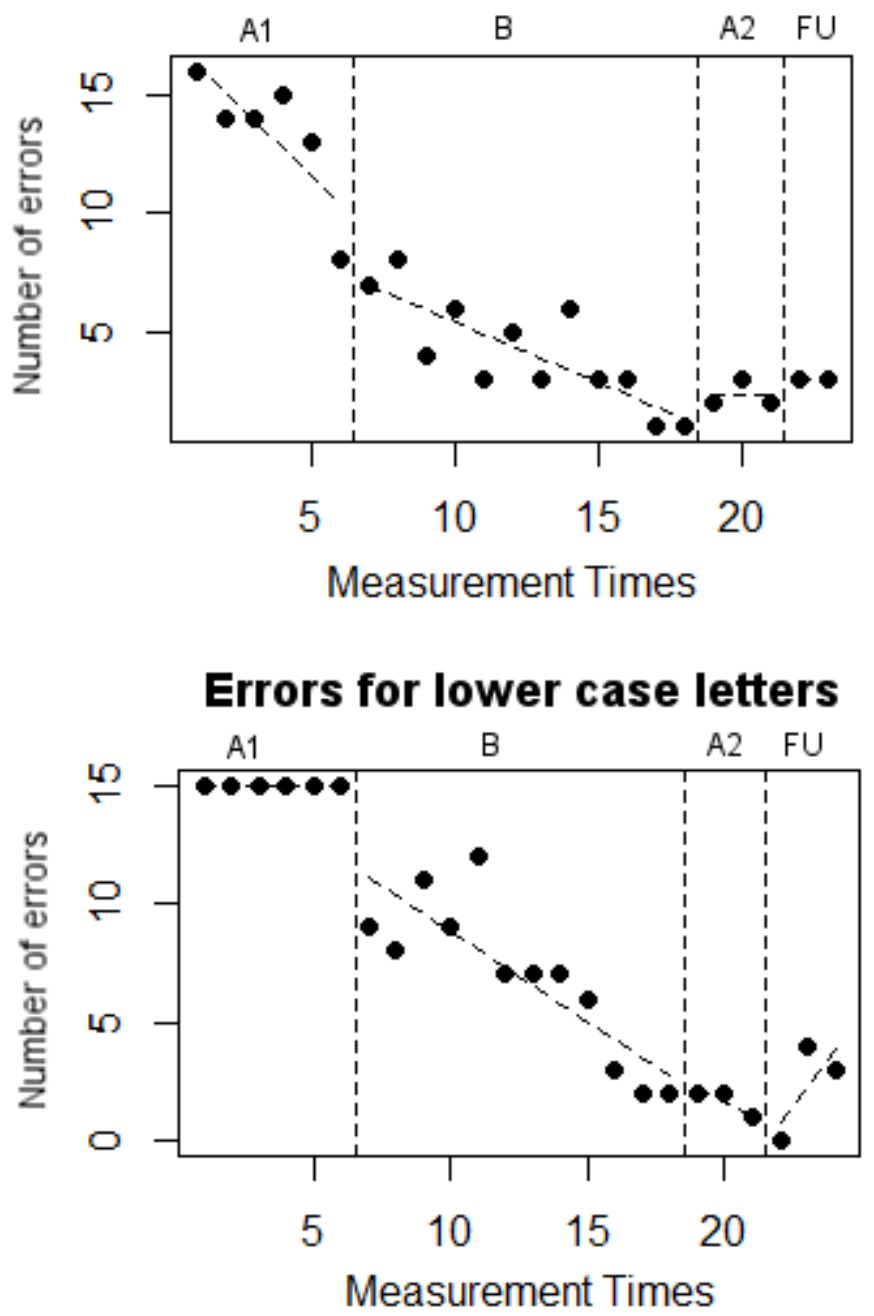

Figure 1. Errors committed for upper case letters (upper panel) and lower case letters (lower panel). Dashed lines represent ordinary least squares regression lines fitted to each phase separately. A1 - initial baseline; B - intervention; A2 - second baseline; FU - follow-up. 

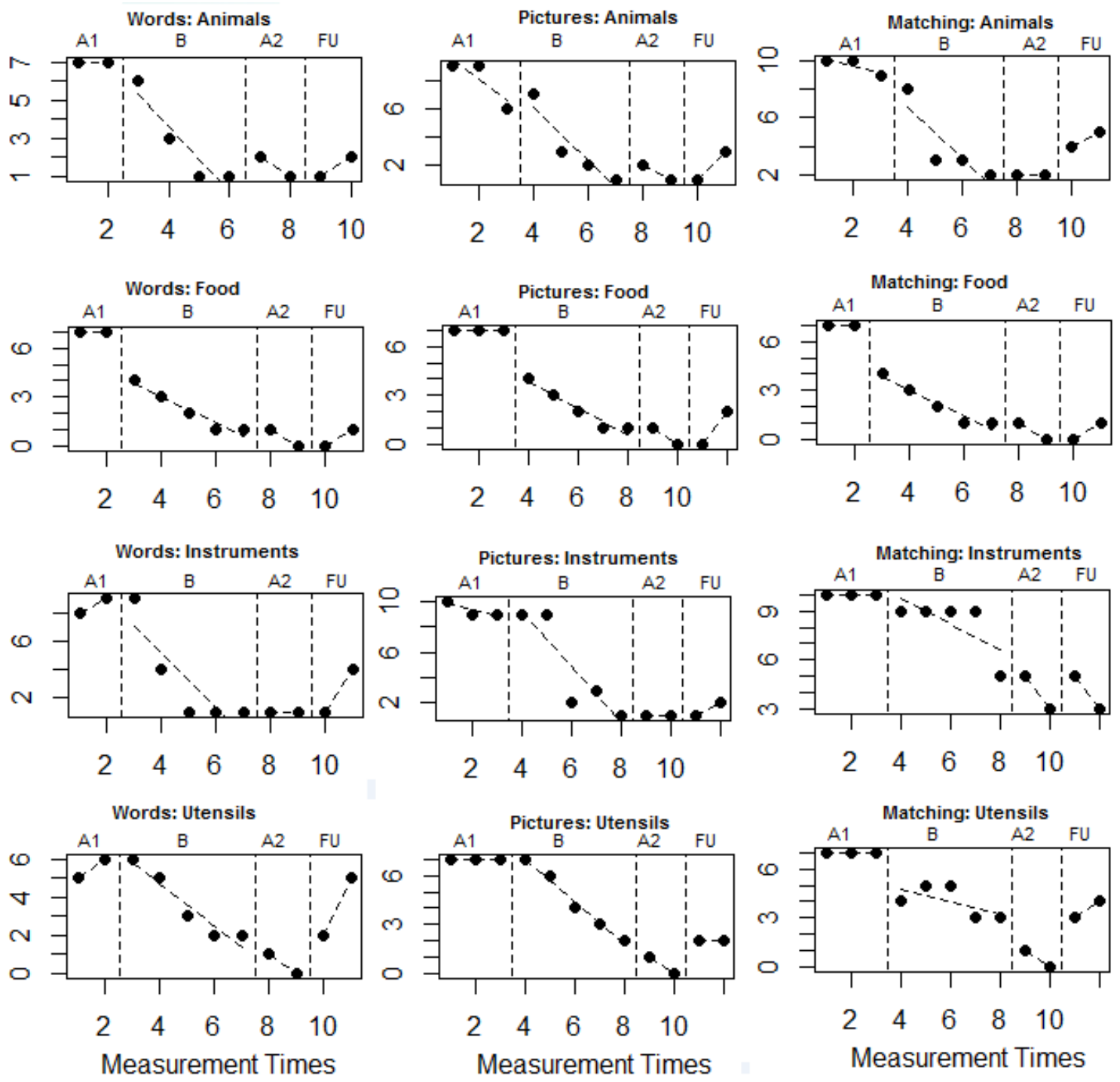

Figure 2. The ordinate in all figures represents the number of errors committed in four different types of words (column 1), pictures (column 2), and matching (column 3). Dashed lines represent ordinary least squares regression lines fitted to each phase separately. A1 initial baseline; B - intervention; A2 - second baseline; FU - follow-up. 
